\section{Need for consensus guidelines to standardise the assessment of germinal centres and other histopathological parameters in salivary gland tissue of patients with primary Sjögren's syndrome}

We have read with great interest the letter to the editor by van Roon et $a l^{1}$ commenting on our paper 'Towards personalised treatment in primary Sjögren's syndrome: baseline parotid histopathology predicts responsiveness to rituximab treatment'. ${ }^{2}$ The authors argue that there is a need for standardisation of the histopathological characteristics of salivary gland tissue of patients with primary Sjögren's syndrome (pSS), in general, and of the presence of germinal centres (GCs), in particular.

We fully agree with van Roon $e t a l^{1}$ and other authors about the need for consensus guidelines to standardise the histopathological evaluation of salivary gland biopsies in patients with pSS. ${ }^{3}$ A standardised scoring system may facilitate prognostication and stratification of patients with pSS and is needed for a valid evaluation of various clinical trials. ${ }^{3}$ In particular, histological definition of GCs in salivary gland tissue is warranted, since these structures can be difficult to detect in diagnostic H\&E-stained tissue sections. Detection of GCs in the periductal lymphoid infiltrates of the salivary glands is clinically relevant, because the presence of these structures is associated with more severe disease. ${ }^{4}$ Importantly, the presence of GCs in minor salivary gland biopsies has been postulated to be a predictor of patients who are at risk of lymphoma development. ${ }^{5} 6$ It has to be mentioned, however, that recently, we were not able to confirm these findings for a larger number of mucosa-associated lymphoid tissue (MALT) lymphomas in parotid glands of patients with pSS (Haacke et al, unpublished data).

In our study, we defined GCs in H\&E-stained sections as lighter areas within the lymphoid infiltrate composed of both lymphoid cells (centrocytes, centroblasts) and cells with a nonlymphoid nature (macrophages and follicular dendritic cells (FDCs)) (figure 1A). ${ }^{1}$ Furthermore, the GCs were scored independently by two experienced pathologists. For the inexperienced eye, GCs may be overlooked, because of their small size, or lighter areas within the infiltrate may erroneously be scored as
GCs, while in fact they represent lymphoepithelial lesions. For proper and easy detection of GCs, also by less-trained persons, additional immunohistochemical staining might be helpful. Therefore, we propose to stain for B-cell lymphoma 6 (Bcl-6) to define and identify GCs. Bcl-6 is a transcription factor expressed at high levels by GC B-cells. Like GCs in peripheral lymphoid organs, GCs in salivary glands of patients with pSS are also consistently positive for Bcl-6. As shown in figure $1 \mathrm{~B}$, staining for Bcl-6 allows the easy and unequivocal detection and scoring of GCs in salivary gland biopsies, both in minor and major (parotid) salivary glands. Implementation of Bcl-6 staining is relatively easy, since it is routinely used in pathology laboratories worldwide for the diagnosis of lymphomas. ${ }^{7}$ Other markers, as proposed by Fisher et $a l^{3}$ and van Roon et al, ${ }^{1}$ are less specific and less suitable to detect GCs in routine diagnostics. For example, activation-induced deaminase, an enzyme essential for the function of GCs B-cells, is expressed only by a minority of GCs B-cells in minor salivary glands of patients with pSS, ${ }^{5}$ which may make GCs harder to detect. The long isoform of CD21 (CD21L) has also been suggested for detection of GCs. CD21L is expressed by FDCs. However, although FDCs are a prerequisite for GC function and development, the presence of these cells does not necessarily imply that GCs are present. Indeed, ectopic lymphoid infiltrates in salivary gland tissue of patients with pSS can contain FDC networks in the absence of GCs. ${ }^{8}$ Staining for the CD21L may therefore result in an overestimation of the number of GCs present in salivary gland tissue.

In our study we observed a relative high proportion of the parotid salivary gland biopsies presented with GCs at baseline$67 \%$ and $68 \%$ of patients in the placebo-treated and rituximabtreated groups, respectively. ${ }^{2}$ These are relatively high percentages compared with the general pSS population, in which approximately one-quarter of the minor salivary gland biopsies exhibit GCs. ${ }^{4}$ The reason for this high baseline characteristic can be attributed to the inclusion criteria of our study. In our study, the patients with pSS were all positive for anti-SSA antibodies and had high systemic activity, as indicated by the relatively high European League against Rheumatism Sjogren's Syndrome Disease Activity Index scores. ${ }^{10}$ Indeed, presence of GCs in minor salivary glands has been associated with more severe disease, including systemic proinflammatory mediators and anti-SSA antibodies. ${ }^{4}$ A second explanation for the high number of GCs at

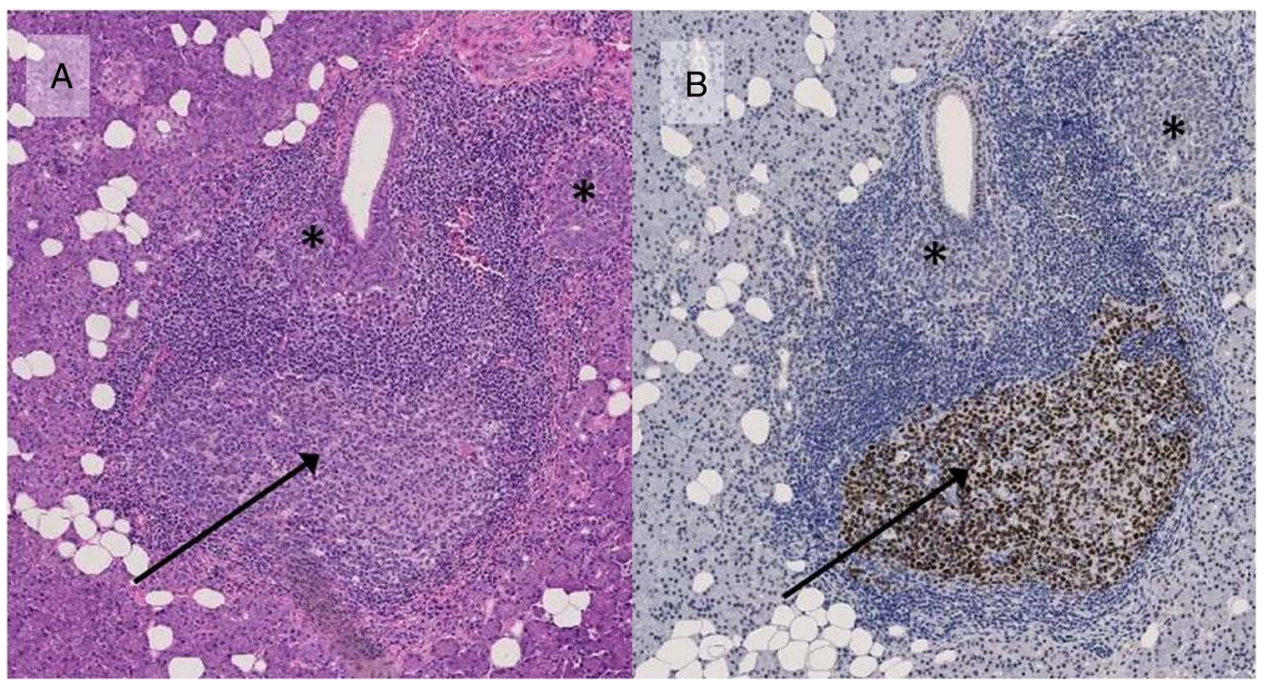

Figure 1 Images showing serial paraffin sections of a parotid gland biopsy of a patient with primary Sjögren's syndrome (pSS) stained with H\&E $(\mathrm{A})$ and for Bcl-6 (B). A clearly visible germinal centre (GC) (indicated with an arrow) is seen in the periductal infiltrate. Asterisks indicate lymphoepithelial lesions. 
baseline might be related to histopathological differences between minor and parotid salivary gland biopsies. Although a previous study in a small cohort of patients with pSS $(n=30)$ did not report a difference in numbers of GCs, ${ }^{11}$ it remains possible that there are more and/or larger GCs in parotid gland biopsies compared with minor salivary glands. Apparently, there is a petition for larger studies focusing on the inherent differences in the histopathological characteristics of parotid and minor salivary gland tissue in both patients with pSS and healthy controls.

In summary, in agreement with van Roon et al, ${ }^{1}$ we would also like to emphasise that there is a need for consensus guidelines to standardise the evaluation of ectopic lymphoid infiltrates and GCs in salivary gland tissue of patients with pSS. The various methods used for automated analysis of several parameters should also be taken into account. ${ }^{12}$ Consensus guidelines will assist the pathologist to correctly identify and quantify histopathological parameters in pSS and contribute to a more accurate prediction of disease progression and personalised treatment, as well as allowing the comparison between study cohorts and different clinical trials.

\section{Konstantina Delli, ${ }^{1}$ Erlin A Haacke, ${ }^{2,3}$ Stephan Ihrler, ${ }^{4}$ Bert van der Vegt, ${ }^{3}$ Arjan Vissink, ${ }^{1}$ Hendrika Bootsma, ${ }^{2}$ Frederik K L Spijkervet, ${ }^{1}$ Frans G M Kroese ${ }^{2}$}

1 Department of Oral and Maxillofacial Surgery, University of Groningen, University Medical Center Groningen, Groningen, The Netherlands

${ }^{2}$ Department of Rheumatology and Clinical Immunology, University of Groningen, University Medical Center Groningen, Groningen, The Netherlands

${ }^{3}$ Department of Pathology and Medical Biology, University of Groningen, University Medical Center Groningen, Groningen, The Netherlands

${ }^{4}$ Laboratory for Dermatohistology and Oral Pathology, Munich, Germany

Correspondence to Dr Konstantina Delli, Department of Oral and Maxillofacial Surgery, University of Groningen, University Medical Center Groningen, Groningen 9713 GZ, The Netherlands; k.delli@umcg.nl

Twitter Follow Konstantina Delli at @KonstantinDelli

Contributors KD, EAK: had the idea for the article, performed the literature search and wrote the article. SI, BvdV, AV, HB, FKLS: drafted the work or revised it critically for important intellectual content. FGMK: had the idea for the article, performed the literature search, wrote the article, drafted the work and revised it critically for important intellectual content.

Competing interests None declared.

Provenance and peer review Commissioned; internally peer reviewed.

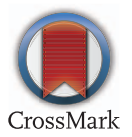

To cite Delli K, Haacke EA, Ihrler S, et al. Ann Rheum Dis 2016;75:e32.

Received 18 March 2016

Accepted 19 March 2016

Published Online First 4 April 2016

\section{Linked}

- http://dx.doi.org/10.1136/annrheumdis-2016-209475

Ann Rheum Dis 2016;75:e32. doi:10.1136/annrheumdis-2016-209480

\section{REFERENCES}

1 Hillen MR, Barone F, van Roon JA, et al. Towards standardisation of histopathological assessments of germinal centres and lymphoid structures in primary Sjögren's syndrome. Ann Rheum Dis 2016;75:e31.

2 Delli K, Haacke EA, Kroese FGM, et al. Towards personalised treatment in primary Sjögren's syndrome: baseline parotid histopathology predicts responsiveness to rituximab treatment. Ann Rheum Dis 2016; Published Online First 12 Jan 2016. doi:10.1136/annrheumdis-2015-208304

3 Fisher BA, Brown RM, Bowman SJ, et al. A review of salivary gland histopathology in primary Sjögren's syndrome with a focus on its potential as a clinical trials biomarker. Ann Rheum Dis 2015;74:1645-50.

4 Risselada AP, Looije MF, Kruize AA, et al. The role of ectopic germinal centers in the immunopathology of primary Sjögren's syndrome: a systematic review. Semin Arthritis Rheum. 2013;42:368-76.

5 Bombardieri M, Barone F, Humby F, et al. Activation-induced cytidine deaminase expression in follicular dendritic cell networks and interfollicular large B cells supports functionality of ectopic lymphoid neogenesis in autoimmune sialoadenitis and MALT lymphoma in Sjögren's syndrome. J Immunol 2007;179:4929-38.

6 Theander $\mathrm{E}$, Vasaitis L, Baecklund $\mathrm{E}$, et al. Lymphoid organisation in labial salivary gland biopsies is a possible predictor for the development of malignant lymphoma in primary Sjögren's syndrome. Ann Rheum Dis 2011;70:1363-8.

7 Tan LH. A practical approach to the understanding and diagnosis of lymphoma: an assessment of the WHO classification based on immunoarchitecture and immuno-ontogenic principles. Pathology 2009;41:305-26.

8 Gommerman JL, Browning JL. Lymphotoxin/light, lymphoid microenvironments and autoimmune disease. Nat Rev Immunol 2003;3:642-55.

9 Jonsson MV, Skarsteine K. Follicular dendritic cells confirm lymphoid organization in the minor salivary glands of primary Sjögren's syndrome. J Oral Pathol Med 2008:37:515-52.

10 Moerman RV, Arends S, Meiners PM, et al. EULAR Sjogren's Syndrome Disease Activity Index (ESSDAI) is sensitive to show efficacy of rituximab treatment in a randomised controlled trial. Ann Rheum Dis 2014;73:472-4.

11 Pijpe J, Kalk WWI, Wal JE et al. Parotid gland biopsy compared with labial biopsy in the diagnosis of patients with primary Sjögren's syndrome. Rheumatology (Oxford) 2007:46:335-41.

12 Delli K, Haacke EA, Kroese FGM, et al. In primary Sjögren's syndrome high absolute numbers and proportions of $B$ cells in parotid glands predict responsiveness to rituximab as defined by ESSDAl, but not by SSRI. Ann Rheum Dis 2016;75:e34. 